\title{
Multiplicative functions of polynomial values in short intervals
}

\author{
by \\ MOHAN NAIR (Glasgow)
}

1. Introduction. Let $d(n)$ denote the divisor function and let $P(n)$ be an irreducible polynomial of degree $g$ with integer coefficients.

In 1952, Erdös [2] showed that there exist constants $c_{1}$ and $c_{2}$, which may depend on $P$, such that

$$
c_{1} x \log x \leq \sum_{n \leq x} d(|P(n)|) \leq c_{2} x \log x
$$

for $x \geq 2$. This result was generalized by Delmer [1] who showed that for any $l \in \mathbb{N}$,

$$
c_{1} x(\log x)^{s} \leq \sum_{n \leq x} d^{l}(|P(n)|) \leq c_{2} x(\log x)^{s}
$$

with $s=2^{l}-1$ and where the $c_{i}$ may depend, in addition, on $l$. The lower bound in Erdős's result is fairly straightforward but the upper bound is a combination of characteristically ingenious ideas.

In 1971, Wolke [6] clarified these ideas and together with several important contributions of his own, showed that any suitable sum of the form $\sum_{n \leq x} f\left(a_{n}\right)$ can be similarly bounded. Here $f$ is any non-negative multiplicative function with $f\left(p^{l}\right) \leq c_{1} l^{c_{2}}, c_{1}, c_{2}$ constants, and $\left\{a_{n}\right\}$ is a sequence of natural numbers with a structure amenable to the sieve method. Applying his results to $f(n)=d(n)$ and $a_{n}=|P(n)|$, he recovered Erdös's result and, indeed, gave several other interesting applications. The very generality of Wolke's results meant that the bounds obtained lacked uniformity with respect to any particular class of sequences $\left\{a_{n}\right\}$.

In 1980, Shiu [5] obtained such a uniformity for the class of arithmetic progressions, i.e. for linear polynomials and refined the Erdős-Wolke method, in this particular case, to include a larger class of multiplicative functions as well as to obtain a short-interval result. He considered the class of non-negative multiplicative functions $f$ which satisfy the weaker conditions $f\left(p^{l}\right) \leq A_{0}^{l}, f(n) \leq A_{1}(\varepsilon) n^{\varepsilon}$ for any $\varepsilon>0$ and constants $A_{0}, A_{1}$. 
He showed that

$$
\sum_{x-y<n \leq x, n \equiv a(\bmod k)} f(n) \ll \frac{y}{\varphi(k)} \frac{1}{\log x} \exp \left(\sum_{p \leq x, p \nmid k} \frac{f(p)}{p}\right)
$$

uniformly for $k \leq y^{1-\beta}$ and $x^{\alpha}<y \leq x$. Here $\varphi(k)$ is Euler's function, $p$ denotes a prime number, $\alpha, \beta \in \mathbb{R}$ with $0<\alpha, \beta<\frac{1}{2}$ and $a, k \in \mathbb{N}$ with $a<k$ and $(a, k)=1$. The implied constant in the $\ll$ notation, although not explicitly stated as such in [5], depends only on $\alpha, \beta, A_{0}$ and the particular function $A_{1}$.

In this paper we extend these ideas further to estimate the general sum $\sum_{x-y<n \leq x} f(|P(n)|)$ while preserving sufficient uniformity with respect to the polynomial $P(n)$ to obtain a bound which implies Shiu's theorem when applied to $P(m)=k m+a,(a, k)=1$ and also, in the general case, extends Erdős's result to polynomials having distinct zeros (not necessarily irreducible) and to $n$ lying in a short interval. The uniformity which we obtain in our theorem has the interesting implication that the apparent generalisation to $n$ lying in an arithmetic progression is, in fact, a corollary of the theorem.

2. Definitions. We define $\mathcal{P}$ to be the class of polynomials $P$ with integer coefficients, of degree $g$, with non-zero discriminant $D$ and having no fixed prime divisors. Let $\varrho(m)$ denote, for $m \in \mathbb{N}$, the number of solutions $n(\bmod m)$ of the congruence $P(n) \equiv 0(\bmod m)$. The condition that $P$ has no fixed prime divisor is equivalent to $\varrho(p)<p$ for all primes $p$. It is well known (see e.g. Nagell [4]) that

(i) $\varrho(a b)=\varrho(a) \varrho(b)$ if $(a, b)=1$,

(ii) $\varrho\left(p^{l}\right) \leq g$ if $p \nmid D$,

(iii) $\varrho(p) \leq g \forall p$, and

(iv) if $p^{\sigma} \| D$ with $\sigma \geq 1$ then

(a) $\varrho\left(p^{l}\right)=\varrho\left(p^{2 \sigma+1}\right)$ if $l>2 \sigma$, and

(b) $\varrho\left(p^{l}\right) \leq p^{l-1} \varrho(p) \quad \forall l \in \mathbb{N}$.

We note that (ii) and (iv) imply that $\varrho\left(p^{l}\right) \leq g p^{2 \sigma}$ and hence that

(v) $\varrho(m) \leq\left(g \bar{D}^{2}\right)^{\omega(m)} \forall m \in \mathbb{N}$,

where the discriminantal factor $\bar{D}$ is defined by

$$
\bar{D}=\prod_{p^{\sigma} \| D, \varrho(p) \neq 0} p^{\sigma} .
$$

We also define the size of $P$, denoted by $\|P\|$, by

$$
\|P\|=\max _{i}\left|a_{i}\right|
$$

where $P(t)=\sum_{0 \leq i \leq g} a_{i} t^{i}$. Note that, $\forall n \in \mathbb{N},|P(n)| \leq(g+1)\|P\| n^{g}$. 
The class $M$ of multiplicative functions which we consider is the same as that in Shiu [5], i.e. $M$ consists of non-negative multiplicative functions which satisfy the following two conditions:

(i) There exists a positive constant $A_{0}$ such that

$$
f\left(p^{l}\right) \leq A_{0}^{l}, \quad \forall \text { primes } p \text { and } l \in \mathbb{N} .
$$

(ii) For every $\varepsilon>0$, there exists a positive constant $A_{1}=A_{1}(\varepsilon)$ such that

$$
f(n) \leq A_{1} n^{\varepsilon}, \quad \forall n \in \mathbb{N} .
$$

We note that the function $\varrho(n)$ itself belongs to $M$ with $A_{0}=g \bar{D}^{2}$ and $A_{1}=A_{1}(g, \bar{D}, \varepsilon)$. For convenience, we also extend the definition of $f$ and put $f(0)=0$.

\section{Notation}

$t, x, y$ and $z$ denote positive real numbers,

$p$ and $q$, with or without subscripts, denote prime numbers,

$a, b, d, g, i, j, k, l, m, n, r$ and $s$, with or without subscripts, denote natural numbers,

$P^{+}(n)$ and $P^{-}(n)$ denote, respectively, the greatest and the least prime factor of $n(\geq 2)$. For technical convenience, we adopt the convention $P^{-}(1)=\infty$ and $P^{+}(1)=1$,

$\Omega(n)$ and $\omega(n)$ denote, as usual, the number of prime factors of $n$ with and without counting multiplicity, respectively,

$\alpha, \beta, \vartheta, \varepsilon$ and $\delta$ denote positive real numbers less than 1 ,

$c, c_{1}, c_{2}, \ldots$ denote positive real constants and

$$
\Psi(x, z)=\sum_{n \leq x, P^{+}(n) \leq z} 1 .
$$

The dependence of the constant implicit in the $\ll$ notation on the polynomial $P$, unless emphasised otherwise, will always be at most on the degree $g$ and on the prime power divisors of the discriminantal factor $\bar{D}$. It may, however, depend on all constants associated with $f$ and with the interval $y$.

4. Results. Our main theorem is the following.

Theorem. Let $f \in M, P \in \mathcal{P}$ and let $\alpha, \delta \in \mathbb{R}$ with $0<\alpha, \delta<1$. For any $x, y \in \mathbb{R}$ with $x, y \geq 2$ and $x^{\alpha} \leq y \leq x$ we have

$$
\sum_{x-y<n \leq x} f(|P(n)|) \ll y \prod_{p \leq x}\left(1-\frac{\varrho(p)}{p}\right) \exp \left(\sum_{p \leq x} \frac{f(p) \varrho(p)}{p}\right)
$$

provided that $x \geq c\|P\|^{\delta}$, where the constant $c$ depends only on $g, \alpha, \delta$ and the function $A_{1}$ associated with $M$. 
The dependence of the constant implicit in the $\ll$ notation on the polynomial $P$ is only on

(i) the degree $g$ and on

(ii) the discriminantal factor $\bar{D}$ mentioned earlier.

It can, of course, depend on $\alpha, \delta$ and the constants associated with the class $M$.

We make the observation that, in general, one would expect an upper bound for the sum considered in our theorem to depend on $\varrho\left(p^{l}\right)$ for all $l \geq 1$. Since our bound depends, explicitly, only on $\varrho(p)$ and it is well known that $\varrho\left(p^{l}\right)$ can be large for primes which divide the discriminant of $P$, it is perhaps to be expected that there is some dependence on $\bar{D}$ in the implicit constant.

We now state the corollary of the theorem which extends the result to arithmetic progressions.

COROLlary. With the same hypotheses as in the Theorem, let, in addition, $a, k \in \mathbb{N}$ with $a \leq k,(k, P(a))=1$ and $k \leq y^{1-\beta}$, where $\beta$ is any constant with $0<\beta<1$. Then

$$
\begin{aligned}
& \quad \sum_{x-y<n \leq x, n \equiv a(\bmod k)} f(|P(n)|) \\
& \ll \frac{y}{k \prod_{p \mid k}(1-\varrho(p) / p)} \prod_{p \leq x}\left(1-\frac{\varrho(p)}{p}\right) \exp \left(\sum_{p \leq x, p \nmid k} \frac{f(p) \varrho(p)}{p}\right)
\end{aligned}
$$

provided that $x \geq c_{1}\|P\|^{\delta}$ where the constant $c_{1}$ depends only on $g, \alpha, \delta$ and the function $A_{1}$ associated with $M$.

The dependence of the implicit constant on the polynomial $P$ is as described in (i) and (ii) in the statement of the Theorem. There is, in addition to those mentioned there, a dependence on $\beta$. The condition $(k, P(a))=1$ ensures that the sequence $P(n), n \equiv a(\bmod k)$ has no fixed prime divisor.

The Corollary applied to $P(n)=n$, so that $D=1,\|P\|=1, \varrho(p)=1$, $\forall p$, recovers Shiu's theorem in its entirety. In fact, all the implications of Shiu's theorem, as mentioned in [5], can now be extended to the polynomial case. We give just one example:

For $r, l \in \mathbb{N}, r \geq 2$, we have

$$
\sum_{x-y<n \leq x, n \equiv a(\bmod k)} d_{r}^{l}(|P(n)|) \ll \frac{y}{k}\left(\frac{\varphi(k)}{k} \log x\right)^{r^{l}-1}
$$

uniformly in $a, k$ and $y$ with $a, k \in \mathbb{N}, a \leq k,(k, P(a))=1, x^{\alpha} \leq y \leq x$ and $k \leq y^{1-\beta}$ provided that $x \gg 1$. Here $\alpha$ and $\beta$ are any real numbers with $0<\alpha, \beta<1$. 
The implicit constants in this example, and only in this example, may depend on $\mathcal{P}, M, r, l, \alpha$ and $\beta$. The function $d_{r}(n)$ is, as usual, the number of ways of writing $n$ as a product of $r$ factors, taking account of ordering.

5. Preliminary lemmas. We shall make use of the following lemmas.

LEMMA 1. Let $G(t) \in \mathbb{Z}[t]$ with degree g. Let $a \in \mathbb{N}$ and let $x, y, z>0$ with $z \leq y \leq x$. Then

$$
\begin{aligned}
\mid\left\{n: x-y<n \leq x, P^{-}(G(n)) \geq z,\right. & (G(n), a)=1\} \mid \\
& \ll_{g} y \prod_{p<z, p \nmid a}\left(1-\frac{\varrho^{\prime}(p)}{p}\right)
\end{aligned}
$$

where $\varrho^{\prime}(p)$ is the number of solutions $m(\bmod p)$ of the congruence $G(m) \equiv$ $0(\bmod p)$.

Proof. This is a straightforward application of Brun's sieve. First note that if $p_{1}$ is any fixed prime divisor of $G$, then either $p_{1} \mid a$ or $p_{1}<z$ implies that the left-hand side of (5.1) is zero and the result is trivially true. Suppose therefore that any such $p_{1}$ satisfies $p_{1} \nmid a$ and $p_{1} \geq z$. We follow the notation of Halberstam and Richert [3] and consider Brun's sieve with

$$
\begin{gathered}
\mathcal{A}=\{G(n): x-y<n \leq x\}, \quad \mathcal{B}=\{p: p<z, p \nmid a, p \text { prime }\}, \\
w(p)= \begin{cases}\varrho^{\prime}(p) & \text { if } p<z, p \nmid a, \\
0 & \text { otherwise. }\end{cases}
\end{gathered}
$$

It is easily checked that $\Omega_{0}$ is satisfied with $A_{0}=g$ and that $\forall p$,

$$
0 \leq \frac{\omega(p)}{p} \leq 1-\frac{1}{g+1}
$$

so that $\Omega_{1}$ holds with $A_{1}=g+1$. Lemma 2.2, p. 52 of [3] implies that $\Omega_{2}(k)$ holds with $k=A_{0}=A_{2}=g$. The condition $R$ in Brun's sieve ([3], p. 68) is trivially satisfied and the result follows.

LEMMA 2. For any $t \geq 2$, we have

$$
\prod_{p<t^{1 / s}}\left(1-\frac{\varrho(p)}{p}\right) \ll_{g} s^{g} \prod_{p<t}\left(1-\frac{\varrho(p)}{p}\right)
$$

uniformly in $s$, provided that $1 \leq s \leq \log t$.

$$
\prod_{p<t^{1 / 2}, p \nmid a}\left(1-\frac{\varrho(p)}{p}\right) \ll_{g}\left(\frac{a}{\varphi(a)}\right)^{g} \prod_{p<t}\left(1-\frac{\varrho(p)}{p}\right) .
$$

(iii) For any $F \in M$,

$$
\sum_{n \leq t} \frac{F(n) \varrho(n)}{n} \ll \exp \left(\sum_{p \leq t} \frac{F(p) \varrho(p)}{p}\right)
$$


where the implicit constant depends on $g, \bar{D}$ and on the constants $A_{0}$ and $A_{1}$ associated with $F$.

Proof. Let $T$ be defined by

$$
T=\prod_{t^{1 / s} \leq p<t}\left(1-\frac{\varrho(p)}{p}\right)^{-1} .
$$

Using the fact that $\varrho(p) \leq \min \{g, p-1\}$, we deduce that

$$
\begin{aligned}
0 & \leq \log T=\sum_{t^{1 / s} \leq p<t}-\log \left(1-\frac{\varrho(p)}{p}\right) \\
& =\sum_{t^{1 / s} \leq p<t, p \leq g}-\log \left(1-\frac{\varrho(p)}{p}\right)+\sum_{t^{1 / s} \leq p<t, p>g}-\log \left(1-\frac{\varrho(p)}{p}\right) \\
& \leq g \sum_{t^{1 / s} \leq p<t, p>g} \frac{1}{p}+O_{g}(1) \leq g \log s+O_{g}(1) .
\end{aligned}
$$

Thus $T \ll_{g} s^{g}$ and (i) follows.

To show (ii), note that

$$
\begin{aligned}
\prod_{p<t^{1 / 2}, p \nmid a}\left(1-\frac{\varrho(p)}{p}\right) & \leq \prod_{g<p<t^{1 / 2}, p \nmid a}\left(1-\frac{\varrho(p)}{p}\right) \\
& \ll g \prod_{p<t^{1 / 2}}\left(1-\frac{\varrho(p)}{p}\right) \prod_{g<p<t^{1 / 2}, p \mid a}\left(1-\frac{\varrho(p)}{p}\right)^{-1} .
\end{aligned}
$$

By (i) and a simple calculation, this is

$$
\ll_{g} \prod_{p<t}\left(1-\frac{\varrho(p)}{p}\right) \prod_{p \mid a}\left(1-\frac{1}{p}\right)^{-g}=\left(\frac{a}{\varphi(a)}\right)^{g} \prod_{p<t}\left(1-\frac{\varrho(p)}{p}\right)
$$

as required.

For (iii), we have

$$
\begin{aligned}
\sum_{n \leq t} \frac{F(n) \varrho(n)}{n} & \leq \prod_{p \leq t}\left(1+\frac{F(p) \varrho(p)}{p}+\sum_{l=2}^{\infty} \frac{F\left(p^{l}\right) \varrho\left(p^{l}\right)}{p^{l}}\right) \\
& \leq \exp \left(\sum_{p \leq t} \frac{F(p) \varrho(p)}{p}+\sum_{p \leq t} \sum_{l \geq 2} \frac{F\left(p^{l}\right) \varrho\left(p^{l}\right)}{p^{l}}\right) .
\end{aligned}
$$

Since $\varrho\left(p^{l}\right) \leq g \bar{D}^{2}$ and $F\left(p^{l}\right) \leq c p^{l / 3}$, we deduce that

$$
\sum_{p \leq t} \sum_{l \geq 2} \frac{F\left(p^{l}\right) \varrho\left(p^{l}\right)}{p^{l}} \ll 1
$$

and (iii) follows. 
Lemma 3. For all $x \geq 3$, we have

$$
\Psi(x, \log x \log \log x) \leq c \exp \left(\frac{3 \log x}{(\log \log x)^{1 / 2}}\right)
$$

for some absolute constant $c$.

Proof. This is Lemma 1 of Shiu [5].

Lemma 4. Let $F \in M$. For any $t \geq 2$, we have

$$
\sum_{\substack{t^{1 / 2} \leq n \leq t \\ P^{+}(n) \leq t^{1 / s}}} \frac{F(n)}{n} \leq c_{1} \exp \left(\sum_{p \leq t} \frac{F(p)}{p}-\frac{1}{10} s \log s\right)
$$

uniformly in $s, 1 \leq s \leq \log t / \log \log t$, where the constant $c_{1}$ depends only on the constants $A_{0}$ and $A_{1}$ associated with $F$.

P r o of. This is essentially Lemma 4 of Shiu [5] with the implicit constant explicitly described and applied with $k=1$.

6. Proof of the Theorem. Let $z=y^{1 / 2}$ and for each $n$ in $(x-y, x]$, write

$$
|P(n)|=a_{n} b_{n}
$$

where if

$$
|P(n)|=p_{1}^{\alpha_{1}} \ldots p_{j}^{\alpha_{j}} p_{j+1}^{\alpha_{j+1}} \ldots p_{l}^{\alpha_{l}}, \quad p_{1}<p_{2}<\ldots<p_{l},
$$

we define

with $j$ chosen such that

$$
a_{n}=p_{1}^{\alpha_{1}} \ldots p_{j}^{\alpha_{j}}
$$

$$
a_{n} \leq z<a_{n} p_{j+1}^{\alpha_{j+1}} .
$$

If no such $j$ exists, define $a_{n}=1$. The associated $b_{n}$ is just defined by $b_{n}=|P(n)| / a_{n}$. Note that $\left(a_{n}, b_{n}\right)=1$ with $P^{-}\left(b_{n}\right)>P^{+}\left(a_{n}\right)$. We write $P^{-}\left(b_{n}\right)$ as $q_{n}$ and divide the set of $n$ in $(x-y, x]$ into four classes:
I: $q_{n} \geq z^{1 / 2}$,
II: $q_{n}<z^{1 / 2}, a_{n} \leq z^{1 / 2}$,
III: $q_{n} \leq \log x \log \log x, a_{n}>z^{1 / 2}$ and
IV: $\log x \log \log x<q_{n}<z^{1 / 2}, a_{n}>z^{1 / 2}$.

We estimate $\sum f(|P(n)|)$ for $n$ belonging to each class in turn. First we have

$$
\sum_{n \in \mathrm{I}} f(|P(n)|) \ll \sum_{a \leq z} f(a) \sum_{x-y<n \leq x}^{(1)} f(b)
$$


where $\sum^{(1)}$ indicates a sum over those $n$ in $(x-y, x]$ such that $a \mid P(n)$, $P^{-}(b) \geq z^{1 / 2}$ and $(a, b)=1$ where $b=|P(n)| / a$. Now

$$
\left(z^{1 / 2}\right)^{\Omega(b)} \leq b=\frac{|P(n)|}{a} \leq \frac{(g+1)\|P\| x^{g}}{a} \ll x^{g+1 / \delta}
$$

so that $\Omega(b) \ll 1$ and hence $f(b) \leq A_{0}^{\Omega(b)} \ll 1$. This implies that the inner sum in $(6.1)$ is

$$
\ll \sum_{x-y<n \leq x}^{(1)} 1 .
$$

We write this sum as

$$
\sum_{r_{i}(\bmod a)} \sum_{\substack{x-y<n \leq x \\ n \equiv r_{i}(\bmod a)}}^{(1)} 1
$$

where $r_{i}$ denote the $\varrho(a)$ solutions of the congruence $P(m) \equiv 0(\bmod a)$. To investigate the inner sum in (6.2), write $n=a m+r_{i}$ and $P\left(r_{i}\right)=a \lambda_{i}, \lambda_{i} \in \mathbb{Z}$. Then $P(n)=P\left(a m+r_{i}\right)=P\left(r_{i}\right)+a m Q\left(a m, r_{i}\right)$ for some $Q\left(a t, r_{i}\right) \in \mathbb{Z}[t]$, of degree $g-1$. The conditions on the inner sum in (6.2) now reduce to

$$
\begin{gathered}
\frac{x-r_{i}}{a}-\frac{y}{a}<m \leq \frac{x-r_{i}}{a}, \\
P^{-}\left(\lambda_{i}+m Q\left(a m+r_{i}\right)\right) \geq z^{1 / 2}, \\
\left(a, \lambda_{i}+m Q\left(a m+r_{i}\right)\right)=1 .
\end{gathered}
$$

Since $y / a \geq z^{1 / 2}$, we can apply Lemma 1 with $G(t)=\lambda_{i}+t Q\left(a t+r_{i}\right)$ to deduce that the inner sum in (6.2) is

$$
\ll \frac{y}{a} \prod_{p<z^{1 / 2}, p \nmid a}\left(1-\frac{\varrho_{1}(p)}{p}\right)
$$

where $\varrho_{1}(p)$ is the number of solutions of the congruence

$$
\lambda_{i}+t Q\left(a t, r_{i}\right) \equiv 0(\bmod p) .
$$

Since $P\left(a t+r_{i}\right)=a\left(\lambda_{i}+t Q\left(a t+r_{i}\right)\right)$, it is easily verified that $\varrho_{1}(p)=\varrho(p)$ for $p \nmid a$, so that $(6.2)$ reduces to

$$
\ll \sum_{r_{i}} \frac{y}{a} \prod_{p<z^{1 / 2}, p \nmid a}\left(1-\frac{\varrho(p)}{p}\right) .
$$

Hence, from (6.1),

$$
\sum_{n \in \mathrm{I}} f(|P(n)|) \ll \sum_{a \leq z} \frac{f(a) y \varrho(a)}{a} \prod_{p<z^{1 / 2}, p \nmid a}\left(1-\frac{\varrho(p)}{p}\right) .
$$


Using Lemma 2(ii), we deduce the bound

$$
\ll y \prod_{p<z}\left(1-\frac{\varrho(p)}{p}\right) \sum_{a \leq z} \frac{f(a) \varrho(a)}{a}\left(\frac{a}{\varphi(a)}\right)^{g} .
$$

We now note that the function $F(a)=f(a)(a / \varphi(a))^{g} \in M$ and appeal to Lemma 2(i), (iii) to deduce

$$
\ll y \prod_{p<x}\left(1-\frac{\varrho(p)}{p}\right) \exp \left(\sum_{p \leq x} \frac{f(p) \varrho(p)}{p}\right)
$$

as required.

We now turn to $n \in \mathrm{II}$ and note that to each such $n$, there corresponds a prime $p$ and an exponent $s$ such that $p^{s} \| P(n), p \leq z^{1 / 2}$ and $p^{s}>z^{1 / 2}$. For each $p \leq z^{1 / 2}$, let $s_{p}$ denote the least integer $s$ with $p^{s}>z^{1 / 2}$. Hence $s_{p} \geq 2$ and $p^{-s_{p}} \leq \min \left(z^{-1 / 2}, p^{-2}\right)$. Thus

$$
\sum_{p \leq z^{1 / 2}} \frac{1}{p^{s_{p}}} \leq \sum_{p \leq z^{1 / 4}} z^{-1 / 2}+\sum_{z^{1 / 4}<p \leq z^{1 / 2}} p^{-2} \ll z^{-1 / 4} .
$$

Hence we deduce that

$$
\begin{aligned}
\sum_{n \in \mathrm{II}} 1 & \leq \sum_{p \leq z^{1 / 2}} \sum_{\substack{x-y<n \leq x \\
p^{s_{p}} \mid P(n)}} 1=\sum_{p \leq z^{1 / 2}} \varrho\left(p^{s_{p}}\right)\left(\frac{y}{p^{s_{p}}}+O(1)\right) \\
& \ll \sum_{p \leq z^{1 / 2}}\left(\frac{y}{p^{s_{p}}}+O(1)\right) \ll \frac{y}{z^{1 / 4}}+z^{1 / 2} \ll y^{7 / 8} .
\end{aligned}
$$

We now show that the $n$ which belong to III are also few in number. For each $n \in$ III, $\exists a, a \mid P(n), z^{1 / 2}<a \leq z$ and $P^{+}(a)<\log x \log \log x$. Hence

$$
\begin{aligned}
& \sum_{n \in \mathrm{III}} 1 \leq \sum_{\substack{z^{1 / 2}<a \leq z \\
P^{+}(a)<\log x \log \log x}} \sum_{\substack{x-y<n \leq x \\
a \mid P(n)}} 1 \\
& =\sum_{\substack{z^{1 / 2}<a \leq z \\
P^{+}(a)<\log x \log \log x}} \varrho(a)\left(\frac{y}{a}+O(1)\right) \ll y \sum_{\substack{z^{1 / 2}<a \leq z \\
P^{+}(a)<\log x \log \log x}} \frac{\varrho(a)}{a} .
\end{aligned}
$$

Now if $z \geq c(g, \bar{D})$ then

$$
\varrho(a) \leq\left(g \bar{D}^{2}\right)^{\omega(a)} \leq\left(g \bar{D}^{2}\right)^{c \log z / \log \log z} \leq z^{1 / 8} .
$$

On the other hand, if $z<c(g, \bar{D})$ then, trivially,

$$
\varrho(a) \leq a<c(g, \bar{D}) \text {. }
$$


Thus (6.3) reduces, by Lemma 3, to

$$
\ll_{g, \bar{D}} \frac{y z^{1 / 8}}{z^{1 / 2}} \Psi(z, \log x \log \log x)+\frac{y}{z^{1 / 2}} \ll y^{7 / 8} .
$$

Thus

$$
\sum_{n \in \mathrm{II}} 1+\sum_{n \in \mathrm{III}} 1 \ll y^{7 / 8}
$$

Now note that for any $\varepsilon_{1}>0$,

$$
f(|P(n)|) \leq A_{1}\left(\varepsilon_{1}\right)|P(n)|^{\varepsilon_{1}} \leq(g+1)^{\varepsilon_{1}} A_{1}\left(\varepsilon_{1}\right)\|P\|^{\varepsilon_{1}} x^{g \varepsilon_{1}} .
$$

Using $x \geq c\|P\|^{\delta}$ with $\varepsilon_{1}$ sufficiently small, we deduce that $f(|P(n)|) \ll$ $y^{1 / 16}$. Hence

$$
\begin{aligned}
\sum_{n \in \mathrm{II}} f(|P(n)|)+\sum_{n \in \mathrm{III}} f(|P(n)|) & \ll y^{15 / 16} \ll \frac{y}{(\log x)^{g}} \\
& \ll y \prod_{p<x}\left(1-\frac{\varrho(p)}{p}\right) \exp \left(\sum_{p \leq x} \frac{f(p) \varrho(p)}{p}\right)
\end{aligned}
$$

as required.

It remains to consider $n$ belonging to class IV:

$$
\sum_{n \in \mathrm{IV}} f(|P(n)|) \ll \sum_{z^{1 / 2}<a \leq z} f(a) \sum_{x-y<n \leq x}^{(2)} f(b)
$$

where $\sum^{(2)}$ indicates a sum over those $n$ in $(x-y, x]$ such that $a \mid P(n)$, $(a, b)=1$ and $\log x \log \log x<P^{-}(b) \leq z^{1 / 2}$ where $b=|P(n)| / a$. We divide the interval for $P^{-}(b)$ into subintervals $\left(z^{1 /(s+1)}, z^{1 / s}\right]$ where

$$
2 \leq s \leq s_{0}:=[\log z / \log (\log x \log \log x)] .
$$

Note that $s_{0} \leq \log z / \log \log z$.

Consider now those $n$ in $\sum^{(2)}$ for which

$$
z^{1 /(s+1)}<P^{-}(b) \leq z^{1 / s}
$$

For such $n$, we have $P^{+}(a) \leq z^{1 / s}$ and also

$$
z^{\Omega(b) /(s+1)} \leq\left(P^{-}(b)\right)^{\Omega(b)} \leq b \leq(g+1)\|P\| x^{g} \ll x^{g+1 / \delta}
$$

so that $\Omega(b) \ll s$. Hence $f(b) \leq A^{s}$ for some $A>0$. Thus

$$
\sum_{n \in \mathrm{IV}} f(|P(n)|) \ll \sum_{s=2}^{s_{0}} A^{s} \sum_{z^{1 / 2}<a \leq z, P^{+}(a) \leq z^{1 / s}} f(a) \sum_{x-y<n \leq x}^{(3)} 1
$$

where $\sum^{(3)}$ indicates a sum over those $n$ in $(x-y, x]$ such that $a \mid P(n)$, 
$(a, b)=1$ and $z^{1 /(s+1)}<P^{-}(b) \leq z^{1 / s}$ where $b=|P(n)| / a$. So denoting by $r_{i}$ the $\varrho(a)$ solutions of $P(m) \equiv 0(\bmod a)$, we have

$$
\sum_{n \in \mathrm{IV}} f(|P(n)|) \ll \sum_{s=2}^{s_{0}} A^{s} \sum_{\substack{z^{1 / 2}<a \leq z \\ P^{+}(a) \leq z^{1 / s}}} f(a) \sum_{r_{i}(\bmod a)} \sum_{\substack{x-y<n \leq x \\ n \equiv r_{i}(\bmod a)}}^{(3)} 1 .
$$

Writing $n=a m+r_{i}$, we deduce, as with the $n$ in class I, that since $z^{1 /(s+1)} \leq$ $y / a$ the inner sum is

$$
\ll \frac{y}{a} \prod_{p<z^{1 /(s+1)}, p \nmid a}\left(1-\frac{\varrho(p)}{p}\right)
$$

and hence

$$
\begin{aligned}
& \sum_{n \in \mathrm{IV}} f(|P(n)|) \ll \sum_{s=2}^{s_{0}} A^{s} \sum_{\substack{z^{1 / 2}<a \leq z \\
P^{+}(a) \leq z^{1 / s}}} \frac{f(a) y \varrho(a)}{a} \prod_{\substack{1 /(s+1) \\
p<z^{1 / a}}}\left(1-\frac{\varrho(p)}{p}\right) \\
& \ll y \sum_{s=2}^{s_{0}} A^{s} \sum_{\substack{1 / 2<a \leq z \\
z^{+/}(a) \leq z^{1 / s}}} \frac{f(a) \varrho(a)}{a} \prod_{p<z^{1 /(s+1)}}\left(1-\frac{\varrho(p)}{p}\right) \prod_{p \mid a}\left(1-\frac{\varrho(p)}{p}\right)^{-1} .
\end{aligned}
$$

We now appeal to Lemma 2(i), twice, together with a simple calculation to deduce the bound

$$
\ll y \prod_{p<x}\left(1-\frac{\varrho(p)}{p}\right) \sum_{s=2}^{s_{0}} A^{s}(s+1)^{g} \sum_{\substack{z^{1 / 2}<a \leq z \\ P^{+}(a) \leq z^{1 / s}}} \frac{f(a) \varrho(a)}{a}\left(\frac{a}{\varphi(a)}\right)^{g} .
$$

Lemma 4 with $F(a)=f(a) \varrho(a)(a / \varphi(a))^{g}$ now implies that the inner sum above is

$$
\ll \exp \left(\sum_{p \leq z} \frac{F(p)}{p}-\frac{1}{10} s \log s\right) \ll \exp \left(\sum_{p \leq x} \frac{f(p) \varrho(p)}{p}-\frac{1}{10} s \log s\right)
$$

so that we finally deduce that

$$
\begin{aligned}
& \sum_{n \in \mathrm{IV}} f(|P(n)|) \\
\ll & y \prod_{p<x}\left(1-\frac{\varrho(p)}{p}\right) \exp \left(\sum_{p \leq x} \frac{f(p) \varrho(p)}{p}\right) \sum_{s \geq 2}(s+1)^{g} A^{s} \exp \left(-\frac{1}{10} s \log s\right) \\
\ll & y \prod_{p<x}\left(1-\frac{\varrho(p)}{p}\right) \exp \left(\sum_{p \leq x} \frac{f(p) \varrho(p)}{p}\right)
\end{aligned}
$$

as required. This completes the proof of the Theorem. 
7. Proof of the Corollary. We denote by $J$ the interval $((x-a-y) / k$, $(x-a) / k]$ and write

$$
\sum_{\substack{x-y<n \leq x \\ n \equiv a(\bmod k)}} f(|P(n)|)=\sum_{m \in J} f(|P(k m+a)|) .
$$

Put $G(m)=P(k m+a)$. We shall apply our theorem to $G(m)$. First note that $\operatorname{deg} G=\operatorname{deg} P=g$. Recall that if $P(t)=a_{g} t^{g}+\ldots+a_{0}, a_{g} \neq 0$, is any polynomial with zeros $\alpha_{1}, \ldots, \alpha_{g}$ in $\mathbb{C}$, then its discriminant $D$ is given by

$$
D=a_{g}^{2 g-2} \prod_{i<j}\left(\alpha_{i}-\alpha_{j}\right)^{2} .
$$

Hence $G(t)$ has zeros $\left(\alpha_{i}-a\right) / k, 1 \leq i \leq g$, and discriminant

$$
D_{1}=\left(a_{g} k^{g}\right)^{2 g-2} \prod_{i<j}\left(\frac{\alpha_{i}-a}{k}-\frac{\alpha_{j}-a}{k}\right)^{2}=k^{g(g-1)} D .
$$

Further, if $\varrho_{1}(d)$ denotes the number of solutions $(\bmod d)$ of the congruence $G(m) \equiv 0(\bmod d)$, it is easily verified that

$$
\begin{aligned}
& \varrho_{1}(p)=0 \quad \text { if } p \mid k \quad(\text { since }(k, P(a))=1) \text { and } \\
& \varrho_{1}\left(p^{\alpha}\right)=\varrho\left(p^{\alpha}\right) \quad \text { if } p \nmid k .
\end{aligned}
$$

We deduce from (7.1) and (7.2) that

$$
\bar{D}_{1}=\prod_{\substack{p^{\sigma} \| D_{1} \\ \varrho_{1}(p) \neq 0}} p^{\sigma}=\prod_{\substack{p^{\sigma} \| D_{1}, p \nmid k \\ \varrho_{1}(p) \neq 0}} p^{\sigma}=\prod_{\substack{p^{\sigma} \| D, p \nmid k \\ \varrho_{1}(p) \neq 0}} p^{\sigma}=\prod_{\substack{p^{\sigma} \| D, p \nmid k \\ \varrho(p) \neq 0}} p^{\sigma} .
$$

Hence $\bar{D}_{1} \mid \bar{D}$ so that the prime power divisors of $\bar{D}_{1}$ are amongst those of $\bar{D}$. Also note that (7.2) implies that $G(m)$ has no fixed prime divisors. It remains to check the other hypotheses of the Theorem. The condition

$$
\left(\frac{y}{k}\right) \geq\left(\frac{x-a}{k}\right)^{\alpha_{1}}
$$

is satisfied for a suitably small $\alpha_{1}>0$ since $k \leq y^{1-\beta}$ and $y \geq x^{\alpha}$. Further, since $\|G\| \leq k^{g}\|P\|$ and $x \geq c_{1}\|P\|^{\delta}$, we have $x \geq c\|G\|^{\delta_{1}}$ for a suitably small $\delta_{1}>0$. The Theorem now yields that

$$
\begin{aligned}
& \sum_{\substack{x-y<n \leq x \\
n \equiv a(\bmod k)}} f(|P(n)|) \\
& \ll \frac{y}{k} \prod_{p<(x-a) / k}\left(1-\frac{\varrho_{1}(p)}{p}\right) \exp \left(\sum_{p \leq(x-a) / k} \frac{f(p) \varrho_{1}(p)}{p}\right) .
\end{aligned}
$$


Using (7.2) we simplify this to

$$
\begin{aligned}
& \ll \frac{y}{k} \prod_{\substack{p<(x-a) / k \\
p \nmid k}}\left(1-\frac{\varrho(p)}{p}\right) \exp \left(\sum_{\substack{p \leq(x-a) / k \\
p \nmid k}} \frac{f(p) \varrho(p)}{p}\right) \\
& \ll \frac{y}{k \prod_{\substack{p<(x-a) / k \\
p \mid k}}(1-\varrho(p) / p)} \prod_{p<(x-a) / k}\left(1-\frac{\varrho(p)}{p}\right) \exp \left(\sum_{\substack{p \leq x \\
p \nmid k}} \frac{f(p) \varrho(p)}{p}\right) \\
& \ll \frac{y}{k \prod_{p<x}(1-\varrho(p) / p)} \prod_{p<(x-a) / k}\left(1-\frac{\varrho(p)}{p}\right) \exp \left(\sum_{\substack{p \leq x \\
p \nmid k}} \frac{f(p) \varrho(p)}{p}\right) .
\end{aligned}
$$

Since $(x-a) / k \gg x^{\beta}$, we can appeal to Lemma 2(i) to finally obtain the bound

$$
\begin{aligned}
& \ll \frac{y}{k \prod_{\substack{p<x \\
p \mid k}}(1-\varrho(p) / p)} \prod_{p<x}\left(1-\frac{\varrho(p)}{p}\right) \exp \left(\sum_{\substack{p \leq x \\
p \nmid k}} \frac{f(p) \varrho(p)}{p}\right) \\
& \ll \frac{y}{k \prod_{p \mid k}(1-\varrho(p) / p)} \prod_{p<x}\left(1-\frac{\varrho(p)}{p}\right) \exp \left(\sum_{\substack{p \leq x \\
p \nmid k}} \frac{f(p) \varrho(p)}{p}\right)
\end{aligned}
$$

as required.

\section{References}

[1] F. Delmer, Sur la somme de diviseurs $\sum_{k \leq x}\{d[f(k)]\}^{s}$, C. R. Acad. Sci. Paris Sér. A-B 272 (1971), A849-A852.

[2] P. Erdős, On the sum $\sum_{k=1}^{x} d(f(k))$, J. London Math. Soc. 27 (1952), 7-15.

[3] H. Halberstam and H.- E. Richert, Sieve Methods, Academic Press, London 1974.

[4] T. Nagell, Introduction to Number Theory, 2nd ed., Chelsea, New York 1964.

[5] P. Shiu, A Brun-Titchmarsh theorem for multiplicative functions, J. Reine Angew. Math. 313 (1980), 161-170.

[6] D. Wolke, Multiplikative Funktionen auf schnell wachsenden Folgen, ibid. 251 (1971), 54-67.

DEPARTMENT OF MATHEMATICS

UNIVERSITY OF GLASGOW

GLASGOW G12 8QW

SCOTLAND, U.K. 